\title{
Composite patch reinforcement of a cracked simply-supported beam traversed by moving mass
}

\author{
M. S Aldlemy ${ }^{1}$, S. A. K Al-jumaili ${ }^{2}$ R. A. M Al-Mamoori 2 T. Ya ${ }^{3,4}$ and R. Alebrahim 3,* \\ ${ }^{1}$ Department of Mechanical Engineering, Collage of Mechanical Engineering Technology, Benghazi-Libya, \\ ${ }^{2}$ Al-Mussaib Technical College, Al-Furat Al-Awsat Technical University, 51009 Bablyon, Iraq, \\ ${ }^{3}$ High Performance Cloud Computing Centre, UTP, 31750 Tronoh, Perak, Malaysia, \\ ${ }^{4}$ Department of Mechanical Engineering, Faculty of Engineering, UTP, 31750 Tronoh, Perak
}

ABSTRACT - In this study dynamic analysis of a metallic beam under travelling mass was investigated. A beam with an edge crack was considered to be reinforced using composite patch. Euler-Bernoulli beam theory was applied to simulate the time-history behavior of the beam under dynamic loading. Crack in the beam was modeled using a rotational spring. Dimension of the composite patch, crack length, stress intensity factor at crack tip and beam deflection are some parameters which were studied in details. Results were validated against those which were found through Finite Element Method.

\author{
ARTICLE HISTORY \\ Revised: $2^{\text {nd }}$ Nov 2019 \\ Accepted: $17^{\text {th }}$ Dec 2019

\section{KEYWORDS} \\ Beam dynamic analysis; \\ edge crack; \\ composite patch; \\ stress intensity factor.
}

\section{INTRODUCTION}

Reinforcement of the beam structures has been addressed by many researchers. Alebrahim et al. [1-4] were used Shape Memory Alloy (SMA) wires to support a damaged beam-like structure under static and dynamic loadings. Patch repairing method is one of the most economical techniques which are used to repair damaged structures. Healing cracked structure using composite patch has increased considerably in recent years [5-7]. Patch is bonded at damaged area to support a structure against different types of external loading. In the study presented by Schubbe and Mall [8], a composite patch was implemented to repair a cracked aluminum panel. Uniaxial tensile test on a prepared specimen (one with a crack and one without crack) revealed that, the maximum stress on the surface of specimen decreases considerably after application of the patch. Moreover, the load carrying capacity of the patched specimen compared to an unpatched one displayed $42 \%$ increment [9]. In the survey which was implemented by Colombi et al. [10], a central cracked steel specimen was reinforced using composite patches. A significant reduction of stress intensity factor was recorded. Decrement of the crack opening displacement was reported as well. In the work presented by Megueni [11] an aged composite patch was applied to repair the cracked metallic structure. A patch of glass/epoxy reduces the mode I stress intensity factor up to $90 \%$. An aluminium plate with central crack was repaired with two sided composite patches under the tensile static and dynamic loading [12]. It was reported that using carbon fiber composite patch may reduce the mode I stress intensity factor by $10 \%$. Furthermore, increasing patch thickness has significant influence on the reduction of stress intensity factor. It is also shown that, increasing adhesive thickness enhances mode I stress intensity factor. In another study Khalili et al. [13] repaired edge-notch steel specimen using polymer composite and smart patches. A plate with single crack was repaired on both sides and it was resulted that patching has major effect on the mechanical characteristics of the cracked plate. The role of the internal layer in contact with the base plate was mentioned as an important parameter in energy absorption. Additionally, non-symmetrical arrangement of the fibers in the patches was reported to show unreliable behavior in all experiments. Optimum ply orientation in a composite patch is studied to repair a cracked plate under biaxial loading. Ply orientation was optimized based on stress intensity factor reduction. Minimum stress intensity factor is reachable if the arrangement of the ply orientation slightly adjusts with maximum principle tensile stress [14]. Reducing mode I stress intensity factor in a cracked plate using Plexiglas patches was investigated by Papadopoulos [15]. It was resulted that, the strength of the cracked plate can be recovered drastically through bonding patch. However, it was notified that, the full recovery through this method is not promising. Dynamic response of a repaired composite cracked beam with bonded patch was accomplished Vaziri and Nayeb-Hashemi [16]. They have emphasized that the elastic modulus of the adhesive layer is as a main factor in deformation and vibration characteristics of the repaired beam. In the weak adhesive elastic modulus, it was remarked that, the adhesive layer displaces under shear loading. The attachment of a repair patch in a unidirectional fiberglass-reinforced epoxy beam can recover the first resonant frequency accurately. An infinite orthotropic cracked sheet was adhesively bonded by a composite patch [17]. The effect of debonding of the patch at crack location was also considered. Reduction of stress intensity factor at crack tip in the presence of the patch was reported. Using piezoelectric patch is a useful method which has been performed to brace the beam and plate structure. Bonding the piezoelectric patches in the cracked area of a beam structure to reduce the deflection and make a healthy structure was implemented by Ariaei et al. [18]. As long as natural frequencies reduce as a result of the crack in the structure, changing the first natural frequency of the flawed structure toward the healthy one was considered as healing 
criteria. A cantilever cracked structure under transverse loading was repaired using piezoelectric patches. It was shown that, using piezoelectric patches can considerably reduce the strain energy density and stress intensity factors at the crack tip. Reducing patch thickness, enlarging the patch length and increasing the numbers of layer were defined as key-points to magnify patching performance. In the study conducted by Liu [19], applying input voltage higher than the required one to close the crack and using too long patch length were not recommended. Dynamic behavior of an actively repaired cantilever beam was analyzed in the study presented by Alaimo et al. [20]. A single-layer piezoelectric patch was implemented in a cracked beam to repair the structure under different voltages. Studies on different input electric loads showed that, the mean value of the obtained stress intensity factors does not depend on the ramp time in response to the electric ramp load and is smaller than that of Heavy-side load. It has been revealed that, the ramp electric load is desirable to the step load despite the longer time which it needs to reach to the suitable condition. Although many researches have been carried out in this area, a few studies have investigated the effect of using composite patch on the stress intensity factor.

This study is focused on the reinforcement of a cracked simply-supported beam through applying composite patches. Crack was considered in the middle of the beam and Euler-Bernoulli beam model was applied. The time-history behavior of the beam was captured numerically using Transfer Matrix Method (TMM). Crack depth, patch length, patch thickness and stress intensity factor at crack tip are some important parameters which were investigated in this study. All coding and programs were carried out in MATLAB software. Numerical achievements were compared and verified against those which were found through FEM.

\section{COMPOSITE PATCH REINFORCEMENT}

In this study a composite patch was implemented to support a cracked steel beam structure. The used patch has composite structure (unidirectional fibre/glass epoxy) and it is assumed that, there is no slippage at the bonding area. It is also assumed that the mass is moving with constant speed and it is always in contact with beam surface. Figure 1 shows the reinforced beam under moving mass schematically.

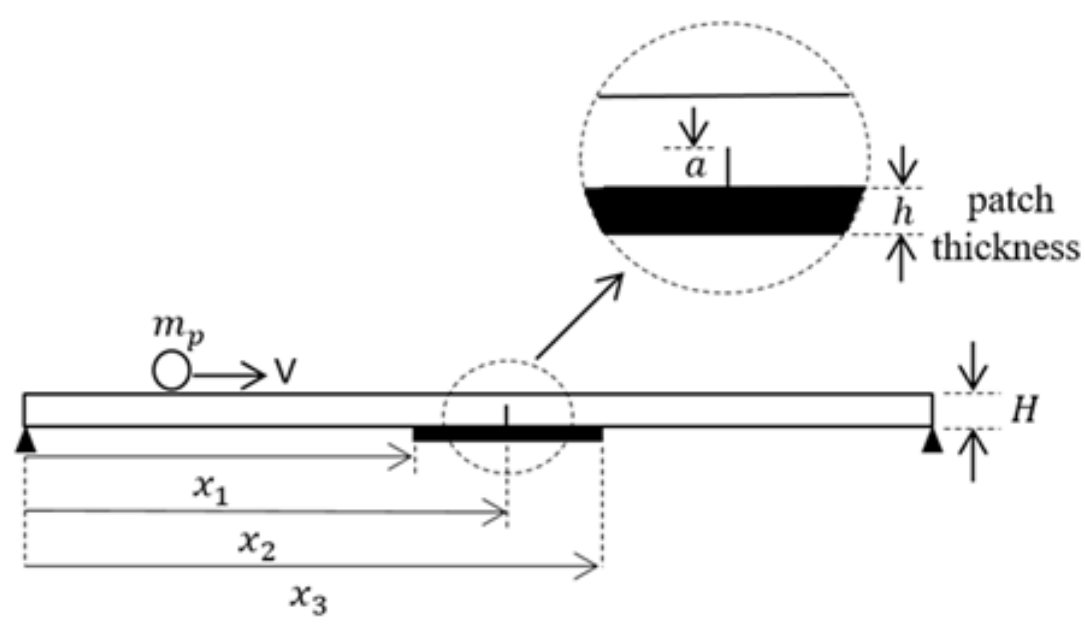

Figure 1. Patched beam with an edge crack located in the beam mid-span.

Euler-Bernoulli beam theory is considered and governing equations for the beam under moving mass is derived as follows:

$$
\begin{gathered}
\rho A_{i} \frac{\partial^{2} y_{i}(x, t)}{\partial t^{2}}+\overline{E I}_{i} \frac{\partial^{4} y_{i}(x, t)}{\partial x^{4}}=P(t) \delta(x-\xi(t)), \\
x_{i-1}<x<x_{i}, \quad i=1,2,3,4
\end{gathered}
$$

where, $y_{i}, \rho A_{i}$ and $\overline{E I}$ are the deflection, longitudinal density and beam rigidity of $i^{\text {th }}$ segment, respectively. The term $\delta(x-\xi(t))$ is the Dirac delta function and $\xi(t)$ denotes the position of moving mass at time $t$. In the case where the mass is moving with constant speed, $\xi(t)$ is equal to $v t$. Where, $v$ is the speed of moving mass. $P(t)$ in Equation. (1) is the force exerted by moving mass and is expressed in the following statement:

$$
P(t)=m_{p}\left(g-\left.\frac{\partial^{2} y(x, t)}{\partial t^{2}}\right|_{x=\xi(t)}\right)
$$


where, $m_{p}$ is the mass of traveler and $g$ is acceleration of gravity. The beam was restrained by simply supported boundary conditions. The state of simply-supported end-conditions is formulated as follows:

$$
y(0, t)=0, \quad y(L, t)=0, \quad \partial^{2} y(0, t) / \partial x^{2}=0, \partial^{2} y(L, t) / \partial x^{2}=0
$$

As presented earlier, crack in the Euler-Bernoulli beam was modelled as rotational spring (Figure 2).

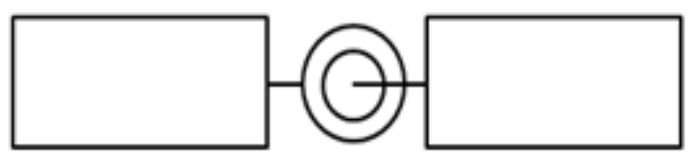

Figure 2. Crack modelling in Euler-Bernoulli beam.

In order to simulate the beam and the installed patch, the length of the beam is divided into 4 segments (Figure 1). Compatibility requirements are then calculated and applied to make the relation between segments. Compatibility requirements for points $x_{1}, x_{2}$ and $x_{3}$ are prepared in the following equations:

Compatibility requirements at point $x_{1}$ :

$$
\begin{aligned}
\frac{\partial^{2} y_{1}\left(x_{1}^{-}, t\right)}{\partial x^{2}} & =\bar{F}_{1} \frac{\partial^{2} y_{2}\left(x_{1}^{+}, t\right)}{\partial x^{2}} \\
\frac{\partial^{3} y_{1}\left(x_{1}^{-}, t\right)}{\partial x^{3}} & =\bar{F}_{1} \frac{\partial^{3} y_{2}\left(x_{1}^{+}, t\right)}{\partial x^{3}} \\
y_{1}\left(x_{1}^{-}, t\right) & =y_{2}\left(x_{1}^{+}, t\right) \\
\frac{\partial y_{1}\left(x_{1}^{-}, t\right)}{\partial x} & =\frac{\partial y_{2}\left(x_{1}^{+}, t\right)}{\partial x}
\end{aligned}
$$

Compatibility requirements at point $x_{2}$ :

$$
\begin{aligned}
& \frac{\partial^{2} y_{2}\left(x_{2}{ }^{-}, t\right)}{\partial x^{2}}=\frac{\partial^{2} y_{3}\left(x_{2}{ }^{+}, t\right)}{\partial x^{2}} \\
& \frac{\partial^{3} y_{2}\left(x_{2}{ }^{-}, t\right)}{\partial x^{3}}=\frac{\partial^{3} y_{3}\left(x_{2}{ }^{+}, t\right)}{\partial x^{3}} \\
& y_{2}\left(x_{2}{ }^{-}, t\right)=y_{3}\left(x_{2}{ }^{+}, t\right) \\
& \frac{\partial y_{2}\left(x_{2}{ }^{-}, t\right)}{\partial x}-\frac{\partial y_{3}\left(x_{2}{ }^{+}, t\right)}{\partial x}=C_{b} H \frac{\partial^{2} y_{3}\left(x_{2}{ }^{+}, t\right)}{\partial x^{2}}
\end{aligned}
$$

Compatibility requirements at point $x_{3}$ :

$$
\begin{aligned}
\frac{\partial^{2} y_{3}\left(x_{3}-, t\right)}{\partial x^{2}} & =\bar{F}_{2} \frac{\partial^{2} y_{4}\left(x_{3}{ }^{+}, t\right)}{\partial x^{2}} \\
\frac{\partial^{3} y_{3}\left(x_{3}{ }^{-}, t\right)}{\partial x^{3}} & =\bar{F}_{2} \frac{\partial^{3} y_{4}\left(x_{3}{ }^{+}, t\right)}{\partial x^{3}}
\end{aligned}
$$




$$
\begin{gathered}
y_{3}\left(x_{3}{ }^{-}, t\right)=y_{4}\left(x_{3}{ }^{+}, t\right) \\
\frac{\partial y_{3}\left(x_{3}{ }^{-}, t\right)}{\partial x}=\frac{\partial y_{4}\left(x_{3}{ }^{+}, t\right)}{\partial x}
\end{gathered}
$$

where, $x^{-}$and $x^{+}$in Equations. (4-15) indicate the location immediately before and after connection points. $\bar{F}_{1}$ and $\bar{F}_{2}$ can be calculated through the following equations:

$$
\begin{aligned}
& \bar{F}_{1}=\frac{\overline{E I}_{2}}{\overline{E I}_{1}} \text { and } \bar{F}_{2}=\frac{\overline{E I}_{4}}{\overline{E I}_{3}} \\
& E_{c}=E_{f} V_{f}+E_{m}\left(1-V_{f}\right)
\end{aligned}
$$

where, $\overline{E I}_{2}$ and $\overline{E I}_{3}$ are the rigidity of the beam with supporting patch (rigidity of segments 2 and 3 in Figure (1)). $\overline{E I}_{1}$ and $\overline{E I}_{4}$ are the rigidity of the beam without supporting patch (rigidity of segments 1 and 4 in Figure (1)). $E_{c}$ in Equation. (16) is the elastic constant of the composite patch. $E_{f}$ and $E_{m}$ are fiber and matrix elastic constant, respectively. $V_{f}$ is the volume fraction of glass fibers. Stress intensity factor at crack tip can be calculated as:

$$
K_{I}=\frac{6 P}{b H^{2}} \sqrt{\pi a} F_{I}(a / \mathrm{H})
$$

where, $P$ is the bending moment at crack tip. $F_{I}$ can be calculated as:

$$
F_{I}\left(\frac{a}{H}\right)=\sqrt{\frac{\tan (\lambda)}{\lambda}}\left[0.923+0.199(1-\sin (\lambda))^{4}\right] / \cos (\lambda), \quad \lambda=\frac{\pi a}{2 H}
$$

$C_{b}$, in Equation. (11) is compliance factor of the crack and it was calculated using fracture mechanics governing equations [21-22]:

$$
C_{b}=\frac{72 I_{b} \pi}{b H^{2}} \int_{0}^{\bar{a}} \bar{\alpha} F_{I}(\bar{\alpha})^{2} d \bar{\alpha}, \quad \bar{a}=a / H
$$

The following procedure was considered to introduce new quantities for formulation:

$$
Y_{i}=\frac{y_{i}}{L}, \quad X=\frac{x}{L}, \quad \bar{T}=\frac{t}{\sqrt{L}}, \quad L_{i}=\frac{l_{i}}{L}, \quad V=\frac{v}{\sqrt{L}} .
$$

Natural frequencies of the beam can be found by solving Equation. (1) for free vibration. It can be achieved by replacing the right hand side of Equation. (1) equal zero.

$$
\rho A_{i} \frac{\partial^{2} Y_{i}(X, \bar{T})}{\partial \bar{T}^{2}}+\frac{\overline{E I}_{i}}{L^{3}} \frac{\partial^{4} Y_{i}(X, \bar{T})}{\partial X^{4}}=0, \quad X_{i-1}<X<X_{i}, i=1,2,3,4
$$

Apply the method of separation of variables $Y_{i}(X, \bar{T})=w_{i}(X) e^{j \omega \bar{T}}$ in Equation. (21), the differential equation of free vibration is derived as follows:

$$
\frac{\partial^{4} w_{i}}{\partial X^{4}}+\lambda_{i}^{4} w_{i}=0 \quad X_{i-1}<X<X_{i}
$$

where, $\lambda_{i}$ represents Eigenvalues of the beam and can be defined as: 


$$
\lambda_{i}^{4}=\frac{\rho_{i} L_{i}^{3}}{E I_{i}} \omega^{2} .
$$

The Eigen solutions of a cracked beam was derived by solving Equation. (22) using the transfer matrix method. The solution for all 4 segments can be written as:

$$
\begin{gathered}
w_{i}(X)=A_{i} \sin \lambda_{i}\left(X-X_{i-1}\right)+B_{i} \cos \lambda_{i}\left(X-X_{i-1}\right)+ \\
C_{i} \sinh \lambda_{i}\left(X-X_{i-1}\right)+D_{i} \cosh \lambda_{i}\left(X-X_{i-1}\right), \quad X_{i-1}<X<X_{i}, \quad i=1,2,3,4 .
\end{gathered}
$$

$A_{i}, B_{i}, C_{i}, D_{i}$ and $\lambda_{i}$ are unknowns which should be determined according to boundary conditions and compatibility equations. Unknown constants of every segment are associated with the next segment through the transfer matrix method. The relation between the first and fourth segments through matrix transfer is shown as follows:

$$
\left[\begin{array}{l}
A_{4} \\
B_{4} \\
C_{4} \\
D_{4}
\end{array}\right]=\boldsymbol{\Gamma}\left[\begin{array}{l}
A_{1} \\
B_{1} \\
C_{1} \\
D_{1}
\end{array}\right] .
$$

In the beam with composite patch, the beam is divided into four segments. Compatibilities requirements should be implemented to make relation between every two segments. Unknown constants of every segment are associated with the next segment through transfer matrix as it is shown in the following statement:

$$
\boldsymbol{\Xi}_{i}\left[\begin{array}{c}
A_{i+1} \\
B_{i+1} \\
C_{i+1} \\
D_{i+1}
\end{array}\right]=\boldsymbol{\Delta}_{i}\left[\begin{array}{c}
A_{i} \\
B_{i} \\
C_{i} \\
D_{i}
\end{array}\right]
$$

where matrixes $\boldsymbol{\Xi}_{i}$ and $\boldsymbol{\Delta}_{\boldsymbol{i}}$ can be defined as:

$$
\begin{gathered}
\boldsymbol{\Xi}_{i}=\left[\begin{array}{llll}
\Xi_{11} & \Xi_{12} & \Xi_{13} & \Xi_{14} \\
\Xi_{21} & \Xi_{22} & \Xi_{23} & \Xi_{24} \\
\Xi_{31} & \Xi_{32} & \Xi_{33} & \Xi_{34} \\
\Xi_{41} & \Xi_{42} & \Xi_{43} & \Xi_{44}
\end{array}\right] \\
\boldsymbol{\Delta}_{i}=\left[\begin{array}{llll}
\Delta_{11} & \Delta_{12} & \Delta_{13} & \Delta_{14} \\
\Delta_{21} & \Delta_{22} & \Delta_{23} & \Delta_{24} \\
\Delta_{31} & \Delta_{32} & \Delta_{33} & \Delta_{34} \\
\Delta_{41} & \Delta_{42} & \Delta_{43} & \Delta_{44}
\end{array}\right] .
\end{gathered}
$$

Matrix components corresponding to every segment of the beam can be calculated as: 
First segment:

$$
\begin{gathered}
\boldsymbol{\Xi}_{1}=\left[\begin{array}{cccc}
\sin \left(\lambda_{1} l_{1}\right) & \cos \left(\lambda_{1} l_{1}\right) & -\sinh \left(\lambda_{1} l_{1}\right) & -\cosh \left(\lambda_{1} l_{1}\right) \\
-\cos \left(\lambda_{1} l_{1}\right) & \sin \left(\lambda_{1} l_{1}\right) & \cosh \left(\lambda_{1} l_{1}\right) & \sinh \left(\lambda_{1} l_{1}\right) \\
\sin \left(\lambda_{1} l_{1}\right) & \cos \left(\lambda_{1} l_{1}\right) & \sinh \left(\lambda_{1} l_{1}\right) & \cosh \left(\lambda_{1} l_{1}\right) \\
\cos \left(\lambda_{1} l_{1}\right) & -\sin \left(\lambda_{1} l_{1}\right) & \cosh \left(\lambda_{1} l_{1}\right) & \sinh \left(\lambda_{1} l_{1}\right)
\end{array}\right] \\
\boldsymbol{\Delta}_{1}=\left[\begin{array}{cccc}
0 & \bar{F}_{1} r_{1}^{2} & 0 & -\bar{F}_{1} r_{1}^{2} \\
-\bar{F}_{1} r_{1}^{3} & 0 & \bar{F}_{1} r_{1}^{3} & 0 \\
0 & 1 & 0 & 1 \\
r_{1} & 0 & r_{1} & 0
\end{array}\right]
\end{gathered}
$$

Second segment:

$$
\begin{gathered}
\boldsymbol{\Xi}_{2}=\left[\begin{array}{cccc}
-\sin \left(\lambda_{2} l_{2}\right) & -\cos \left(\lambda_{2} l_{2}\right) & \sinh \left(\lambda_{2} l_{2}\right) & \cosh \left(\lambda_{2} l_{2}\right) \\
-\cos \left(\lambda_{2} l_{2}\right) & \sin \left(\lambda_{2} l_{2}\right) & \cosh \left(\lambda_{2} l_{2}\right) & \sinh \left(\lambda_{2} l_{2}\right) \\
\sin \left(\lambda_{2} l_{2}\right) & \cos \left(\lambda_{2} l_{2}\right) & \sinh \left(\lambda_{2} l_{2}\right) & \cosh \left(\lambda_{2} l_{2}\right) \\
\cos \left(\lambda_{2} l_{2}\right) & -\sin \left(\lambda_{2} l_{2}\right) & \cosh \left(\lambda_{2} l_{2}\right) & \sinh \left(\lambda_{2} l_{2}\right)
\end{array}\right] \\
\boldsymbol{\Delta}_{2}=\left[\begin{array}{cccc}
0 & -r_{2}^{2} & 0 & r_{2}^{2} \\
-r_{2}^{3} & 0 & r_{2}^{3} & 0 \\
0 & 1 & 0 & 1 \\
1 & C_{b} r_{2} \lambda_{3} & 1 & -C_{b} r_{2} \lambda_{3}
\end{array}\right]
\end{gathered}
$$

Third segment:

$$
\begin{gathered}
\boldsymbol{\Xi}_{3}=\left[\begin{array}{cccc}
\sin \left(\lambda_{3} l_{3}\right) & \cos \left(\lambda_{3} l_{3}\right) & -\sinh \left(\lambda_{3} l_{3}\right) & -\cosh \left(\lambda_{3} l_{3}\right) \\
-\cos \left(\lambda_{3} l_{3}\right) & \sin \left(\lambda_{3} l_{3}\right) & \cosh \left(\lambda_{3} l_{3}\right) & \sinh \left(\lambda_{3} l_{3}\right) \\
\sin \left(\lambda_{3} l_{3}\right) & \cos \left(\lambda_{3} l_{3}\right) & \sinh \left(\lambda_{3} l_{3}\right) & \cosh \left(\lambda_{3} l_{3}\right) \\
\cos \left(\lambda_{3} l_{3}\right) & -\sin \left(\lambda_{3} l_{3}\right) & \cosh \left(\lambda_{3} l_{3}\right) & \sinh \left(\lambda_{3} l_{3}\right)
\end{array}\right] \\
\boldsymbol{\Delta}_{3}=\left[\begin{array}{cccc}
0 & \bar{F}_{2} r_{3}^{2} & 0 & -\bar{F}_{2} r_{3}^{2} \\
-\bar{F}_{2} r_{3}^{3} & 0 & \bar{F}_{2} r_{3}^{3} & 0 \\
0 & 1 & 0 & 1 \\
r_{3} & 0 & r_{3} & 0
\end{array}\right]
\end{gathered}
$$

where $l_{1}, l_{2}$ and $l_{3}$ are the length of first, second and third segments, respectively. $r_{1}, r_{2}$ and $r_{3}$ are defined as follows:

$$
r_{1}=\frac{\lambda_{2}}{\lambda_{1}}, r_{2}=\frac{\lambda_{3}}{\lambda_{2}} \text { and } r_{3}=\frac{\lambda_{4}}{\lambda_{3}}
$$

The relation between first and fourth segment can be defined as:

$$
\left[\begin{array}{l}
A_{4} \\
B_{4} \\
C_{4} \\
D_{4}
\end{array}\right]=\boldsymbol{\Gamma}\left[\begin{array}{l}
A_{1} \\
B_{1} \\
C_{1} \\
D_{1}
\end{array}\right]
$$


where, $\boldsymbol{\Gamma}$ can be calculated as follows:

$$
\boldsymbol{\Gamma}=\left(\Delta_{3}^{-1}\right)\left(\boldsymbol{\Xi}_{3}\right)\left(\Delta_{2}^{-1}\right)\left(\boldsymbol{\Xi}_{2}\right)\left(\Delta_{1}^{-1}\right)\left(\boldsymbol{\Xi}_{1}\right) .
$$

By applying boundary conditions in Equation. (36), the following results were obtained:

$$
\begin{gathered}
B_{1}=0, \quad D_{1}=0 \\
\boldsymbol{B}\left[\begin{array}{l}
A_{4} \\
B_{4} \\
C_{4} \\
D_{4}
\end{array}\right]=\left[\begin{array}{l}
0 \\
0
\end{array}\right],
\end{gathered}
$$

where $\boldsymbol{B}$ was defined as:

$$
\boldsymbol{B}_{2 \times 4}=\left[\begin{array}{cccc}
\sin \lambda_{4} l_{4} & \cos \lambda_{4} l_{4} & \sinh \lambda_{4} l_{4} & \cosh \lambda_{4} l_{4} \\
-\sin \lambda_{4} l_{4} & -\cos \lambda_{4} l_{4} & \sinh \lambda_{4} l_{4} & \cosh \lambda_{4} l_{4}
\end{array}\right]
$$

From Equations. (36-40) it can be concluded that:

$$
\boldsymbol{B . \Gamma}\left[\begin{array}{l}
A_{1} \\
B_{1} \\
C_{1} \\
D_{1}
\end{array}\right]=\boldsymbol{R}\left[\begin{array}{l}
A_{1} \\
B_{1} \\
C_{1} \\
D_{1}
\end{array}\right]=\left[\begin{array}{l}
0 \\
0
\end{array}\right],
$$

According to Equation. (41), $B_{1}$ and $D_{1}$ are zero; by substituting these values in Equation. (41), matrix $\boldsymbol{R}$ would reduce to a square matrix. The determinant of the resultant matrix gives the characteristic equation. Eigenvalues of the beam may be attained through solving the characteristic equation. The forced response of Equation. (1) was investigated using the modal expansion theory. Rewriting Equation. (1) in non-dimensional form:

$$
\rho A_{i} \frac{\partial^{2} Y_{i}}{\partial \bar{T}^{2}}+\frac{\overline{E I}_{i}}{L^{3}} \frac{\partial^{4} Y_{i}}{\partial X^{4}}=P(\bar{T}) \delta(X-V \bar{T}) \quad X_{i-1}<X<X_{i},
$$

According to the modal expansion theory, the forced response $Y$ can be mentioned as:

$$
Y(X, \bar{T})=\sum_{k=1}^{N} w_{k}(X) T_{k}(\bar{T})
$$

In Equation. (43), $w_{k}(X)$ and $T_{k}(\bar{T})$ are normalized Eigen functions and generalized coordinates of the cracked beam, respectively. $N$, is the number of Eigen functions used to approximate the solution. Substituting Equation. (43) in Equation. (42), multiplying both sides by $w_{j}(X)$ and then integrating from 0 to 1 result in;

$$
\sum_{k=1}^{N}\left(\ddot{T}_{k}(\bar{T})+\omega_{k}^{2} T_{k}(\bar{T})\right) \int_{0}^{1} w_{j}(X) w_{k}(X) d X=\int_{0}^{1} \frac{P(\bar{T})}{\rho A} \delta(X-V \bar{T}) w_{j}(X) d X
$$

Applying the orthogonality principle of Eigen functions, Equation. (44) leads to:

$$
\left(\ddot{T}_{k}(\bar{T})+\omega_{k}^{2} T_{k}(\bar{T})\right)=\int_{0}^{1} \frac{P(\bar{T})}{\rho A} \delta(X-V \bar{T}) w_{k}(X) d X=
$$




$$
\frac{P(\bar{T})}{\rho A} w_{k}(V \bar{T})=\frac{M}{\rho A} w_{k}(V \bar{T})\left(g-\sum_{k=1}^{N} w_{k}(V \bar{T}) \ddot{T}_{k}(\bar{T})\right)=Q_{k}(\bar{T})
$$

The right hand side of Equation. (45) is the function of $T_{k}(\bar{T})$, which shows a set of coupled second order differential equations. Generalized coordinates can be calculated by solving Equation. (45) for $T_{k}(\bar{T})$. The deflection of the beam then can be found using Equation. (43).

\section{RESULTS AND DISCUSSION}

The reinforcement of a beam with a crack in the middle was simulated and analysed. A fiberglass composite patch was bonded in the crack area to support the beam against transverse loading. Mechanical properties of the beam and moving mass are as listed in Table 1.

Table 1. Mechanical properties of the beam and moving mass

\begin{tabular}{cccc}
\hline \multicolumn{3}{c}{ Mechanical Properties of the Beam and Moving Mass } \\
\hline $\mathrm{L}=1 \mathrm{~m}$ & $E_{b}=2100 \mathrm{GPa}$ & $\mathrm{v}=40 \mathrm{~m} / \mathrm{s}$ & $\rho=7860 \mathrm{kgm}^{-3}$ \\
$\mathrm{H}=0.02 \mathrm{~m}$ & $\mathrm{a} / \mathrm{H}=0.5$ & $\mathrm{~b}=0.02 \mathrm{~m}$ & $m_{p}=0.2 \rho A L$ \\
$E_{f}=\mathbf{8 0} \mathbf{~ G P a}$ & $E_{m}=2.79 \mathrm{GPa}$ & $V=70 \%$ & $\mathrm{~h}=0.01 \mathrm{~m}$ \\
\hline
\end{tabular}

\section{Natural Frequency Analysis of Reinforced Beam}

Natural frequencies of cracked beam with a composite patch were obtained. Figure 3 shows the changing of first, second and third natural frequencies of the reinforced beam. The natural frequencies of the intact beam (with no crack and no patch) according to Figure 3 are listed as $46.95,187.64$ and $421.91 \mathrm{~Hz}$. These results are in close agreement with those which are addressed in reference [18]. It can be observed that by increasing the crack depth the first and third natural frequencies have decreased considerably. However, the second natural frequency has not changed during this process. It might be due to the type of the second mode shape. The mode shapes of an intact beam are brought in Figure 4. According to the second mode shape in Figure 4, the mid-span point is motionless. It can be observed that only first and third mode shape effect on the beam deflection in the mid-span.

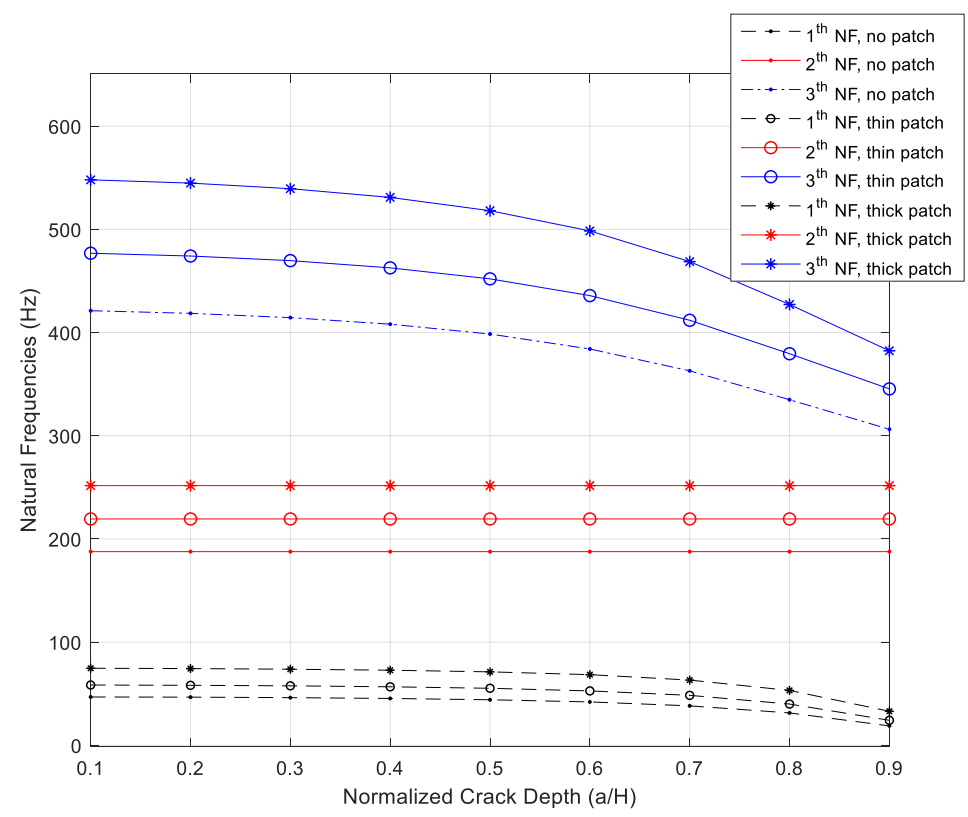

Figure 3. Changing of first, second and third natural frequencies versus crack depth. 


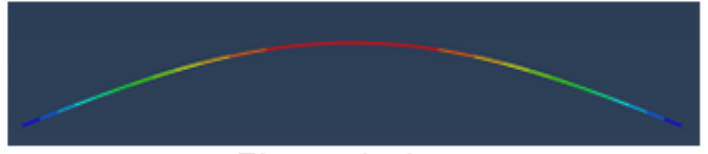

First mode shape

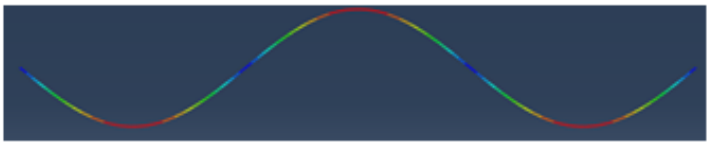

Third mode shape

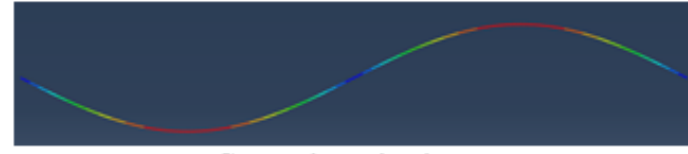

Second mode shape

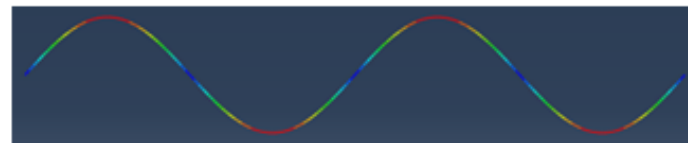

Forth mode shape

Figure 4. First four mode shape of the beam.

It can be found in Figure 3 that all natural frequencies have improved greatly using composite patch (the length of the patch in this example is $60 \mathrm{~cm}$ ). As it is expected, the thick patch has more influence on the enhancement of natural frequencies. The thickness of the thin and thick patches is 0.01 and $0.02 \mathrm{~m}$, respectively. The optimum length of the patch for every crack depth is addressed in Figure 5. These optimum lengths were found based on the recovery which was observed in the first and third natural frequencies. Using these optimum patch lengths for corresponding crack depths, one may obtain the deflection of a healthy beam at mid-span. It should be noted that according to the mode shapes plotted in Figure 4, second and forth natural frequencies do not effect on the deflection of the mid-span of the beam. As it is noted before the main focus of current study is to recover the behavior of mid-span of the beam under moving mass. In order to recover the deflection of other points in the beam structure, the role of the second and forth natural frequencies should be considered as well. The optimization of the patch length was carried out for two different patch thicknesses (see Figure 5). The optimized patch length increases rapidly by growing of the crack depth. It was found that the thin patch $(\mathrm{h}=0.01 \mathrm{~m})$ is not able to perfectly recover the beam behavior at mid-span for $\mathrm{a} / \mathrm{H}>0.7$. However, the thick patch $(\mathrm{h}=0.02 \mathrm{~m})$ could be applied effectively for $\mathrm{a} / \mathrm{H}>0.7$.

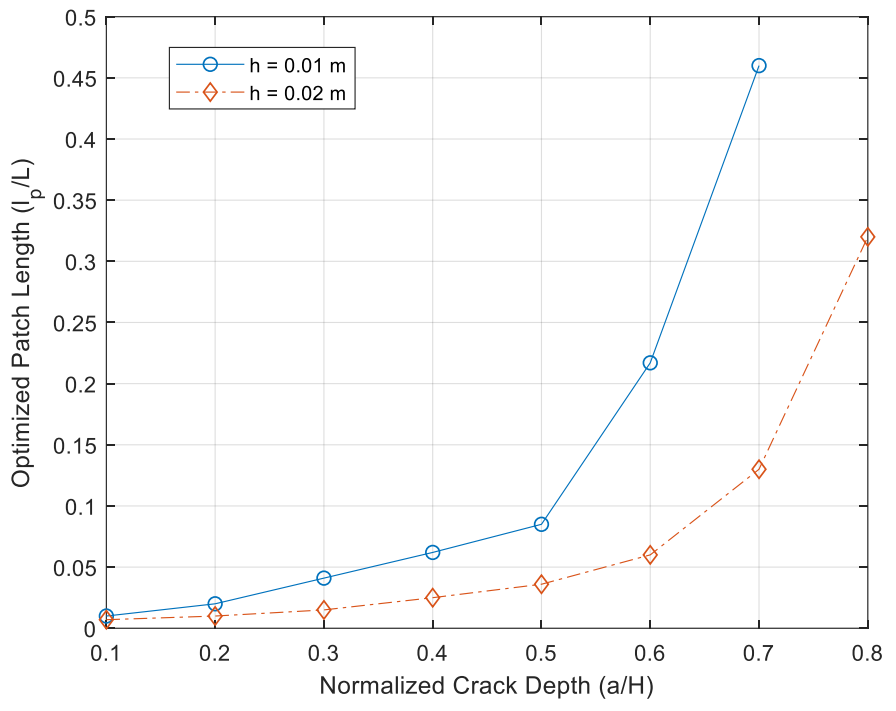

Figure 5. Optimized patch length versus crack depth for different patch thicknesses.

Time history of the beam deflection as the result of moving mass were obtained and plotted in Figure 6 . It is clear that using patch with optimized length $(l p / L=0.46)$ completely improves the deflection of the damaged beam $(a / H=0.7)$. $T_{0}$ in Figures 6 is defined as $T_{0}=L / v$. 


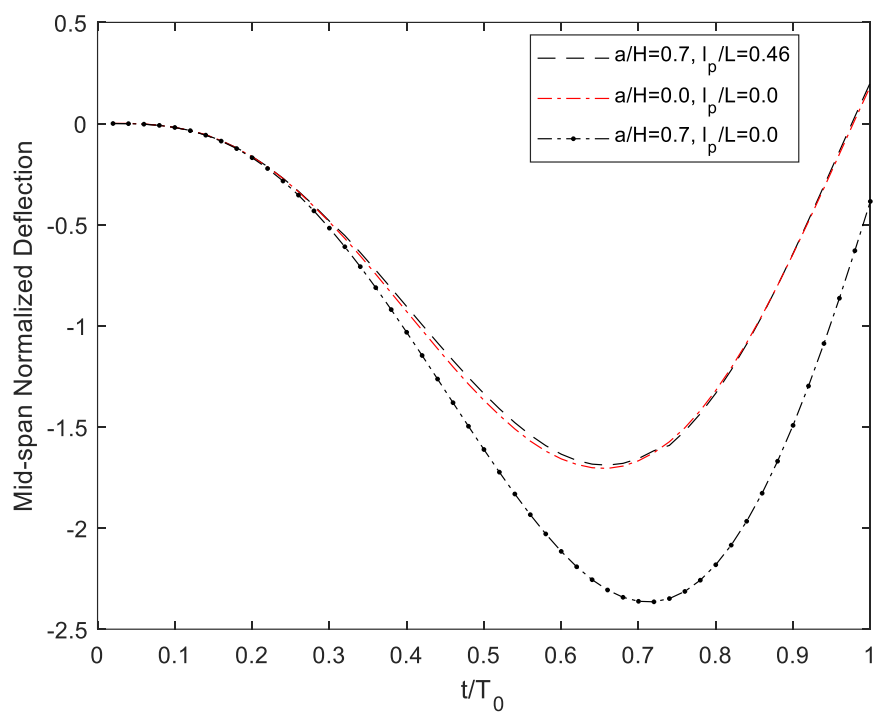

Figure 6. Recovery of mid-span displacement using composite patch, $\boldsymbol{T}_{\mathbf{0}}=\boldsymbol{L} / \boldsymbol{v}$.

The mid-span deflections for thin and thick cracks as a function of patch length are plotted in Figure 7. The crack depth in this case study is considered as $70 \%$ of the beam thickness. It can be perceived from Figure 7 that for the normalized patch length between 0.1 and 0.7 the patch has great influence on the reduction of beam deflection. However, exceeding the patch length more than $70 \%$ of the beam's length does not produce efficient results for both thin and thick patches. As it is shown in Figure 7, the deflection of the beam does not reduce and remains almost constant for $l p / L>$ 0.7. Using thick patch has made a great shift in the results of mid-span deflection. Doubling the patch thickness from 0.01 to $0.02 \mathrm{~m}$ has decreased the deflection 2 to more than 4 units.

\section{Stress Intensity Factor in Reinforced Patched Beam}

Stress intensity factors for different crack depths were calculated and plotted. The stress intensity factor in crack tip was slightly decreased by using a composite patch. Figure 8 shows the changing of the SIF according to the patch length. When the patch length is more than $70 \%$ of the beam's length, the SIF behavior is almost constant. Using effective composite patch length avoids crack from opening and decreases stress intensity factor at the crack tip. According to Figure 8, thin and thick patches have reduced SIF near $47 \%$ and $75 \%$, respectively. Changing the SIF as function of crack depth is depicted in Figure 9. Using the optimized crack length, the improved SIF of each crack depth was calculated. The percentage of SIF Reduction for all crack depths is almost the same and it is $47 \%$ and $75 \%$ for thin and thick cracks, respectively.

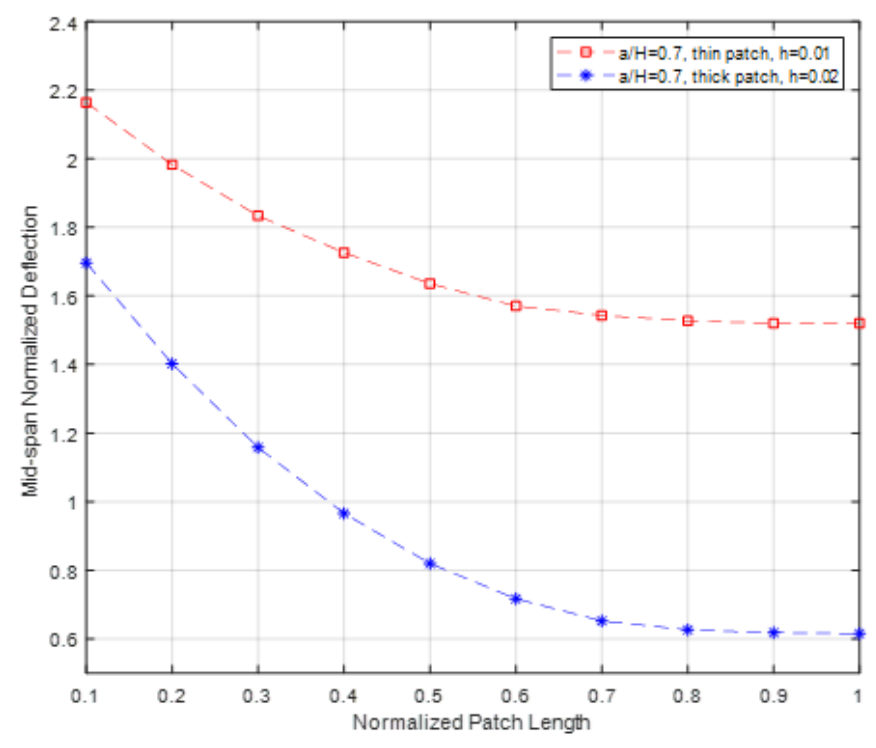

Figure 7. Maximum mid-span deflection versus normalized patch length, $\boldsymbol{l}_{\boldsymbol{p}}$ and $\boldsymbol{L}$ are the length of the patch and beam, respectively. 


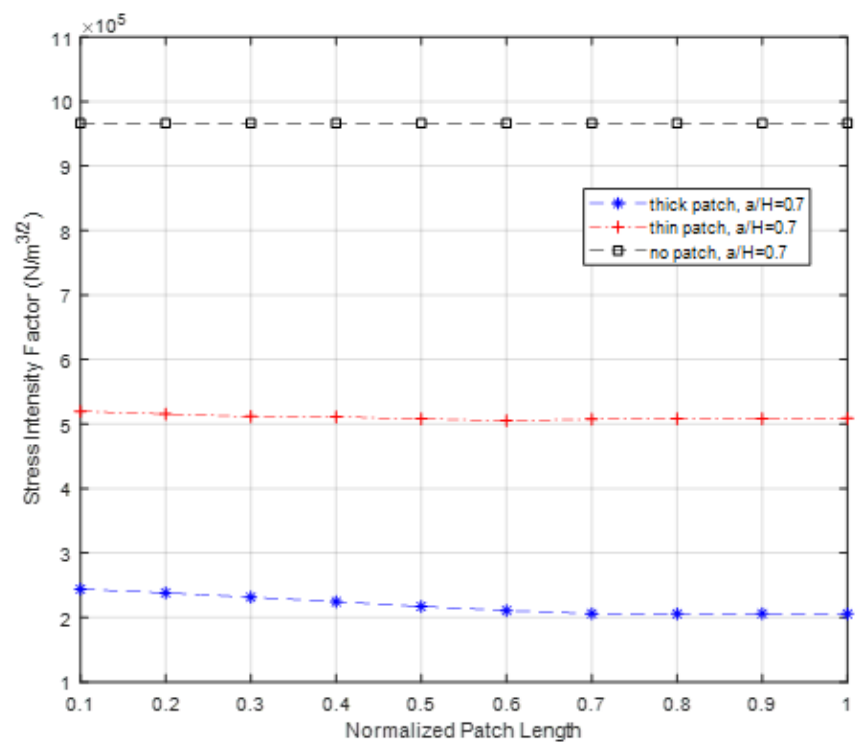

Figure 8. Changing of stress intensity factor by increasing patch length.

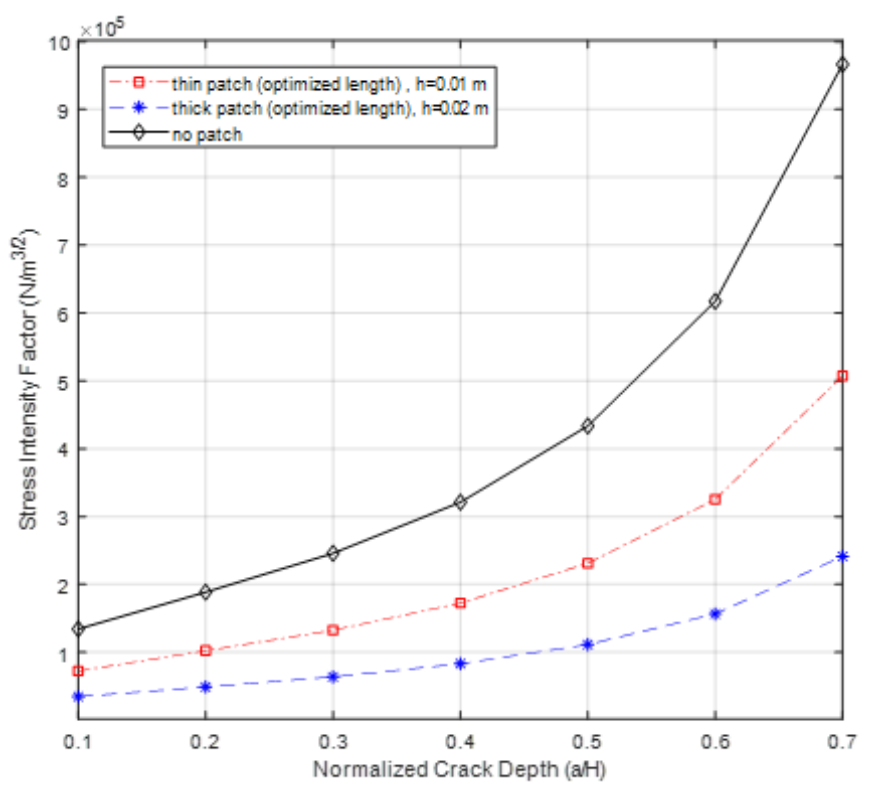

Figure 9. Stress intensity factor recovery for various crack depths.

\section{Finite Element Method}

In order to validate the results, the finite element method was used. As long as the dynamic problem is in concern, Abaqus/Explicit was implemented. A beam with a crack in the middle was modeled in the Abaqus/Explicit and results were compared with those which were found through TMM. The type of element which is applied in this model is Eightnode brick element (C3D8R) and the number of elements is 6250. Figure 10 shows the beam with the bonded patch under moving mass. All mechanical properties of the beam and moving mass obey those which were presented in Table 1 . The mass was simulated as a cubic slider. Frictionless contact was defined between the side of a moving cube and the beam surface. Mid-span deflection results which were obtained through TMM, are verified against FEM findings (see Figure 11). Comparison of results for different crack lengths confirms the accuracy of the applied method. Results verification for using patch was also checked in Figure 12. It can be observed that applying optimized length of the patch $l p / L=$ 0.04 has recovered the deflection of cracked beam $(a / H=0.5)$ in both TMM and FEM methods. 


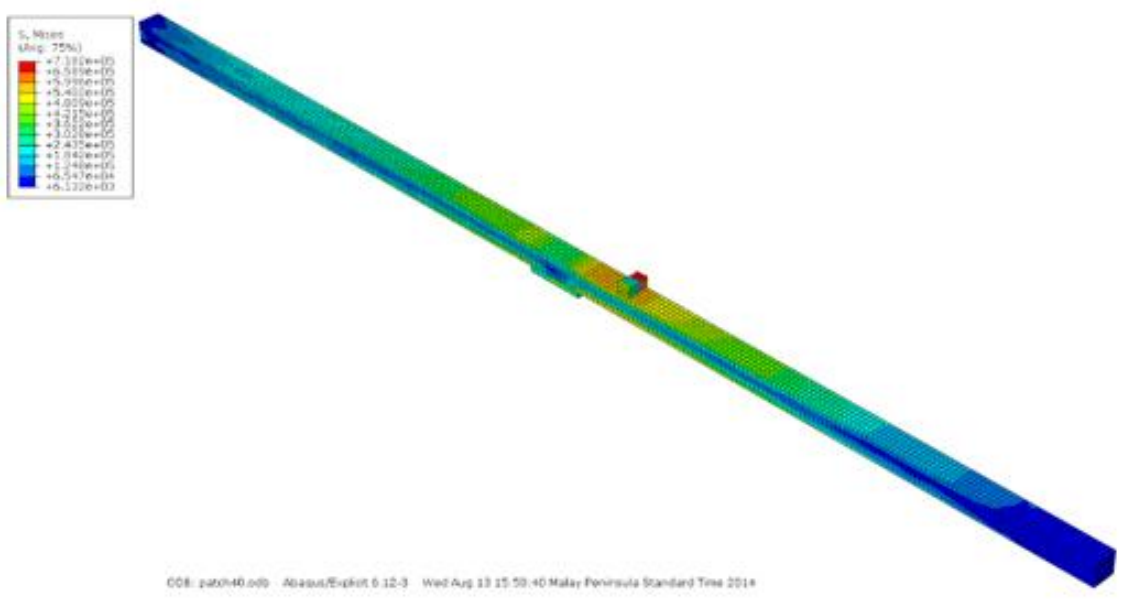

Figure 10. Composite patch reinforced cracked beam under moving mass.

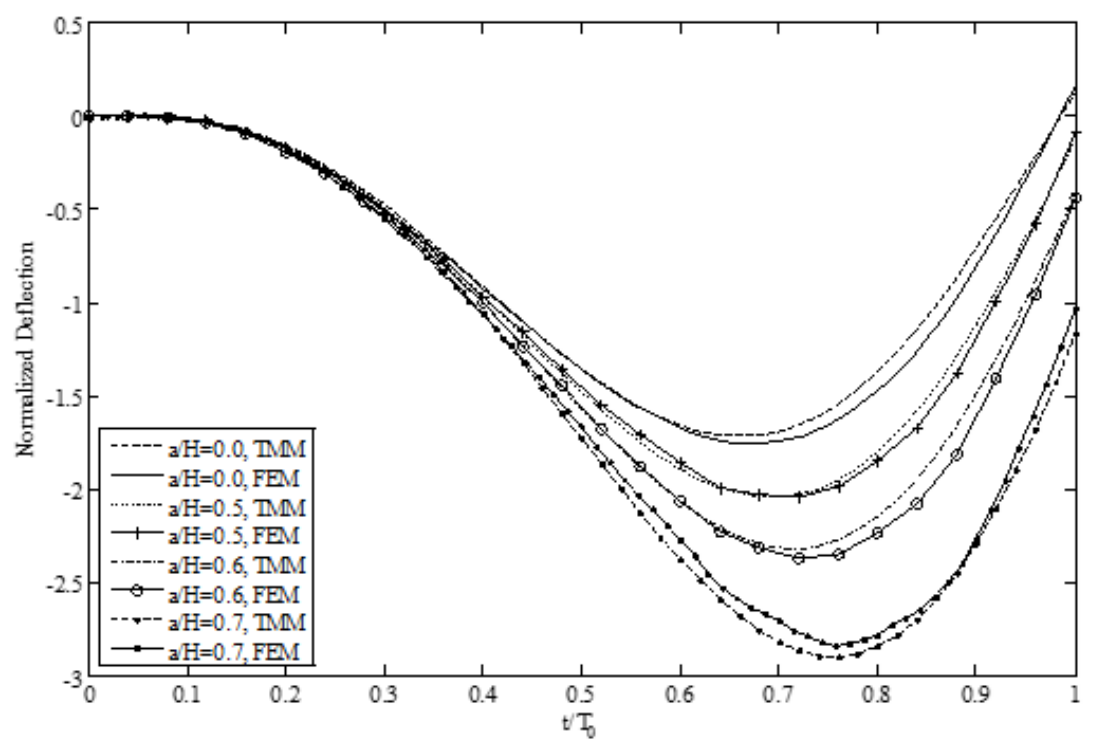

Figure 11. Normalized deflection of the beam using TMM and FEM.

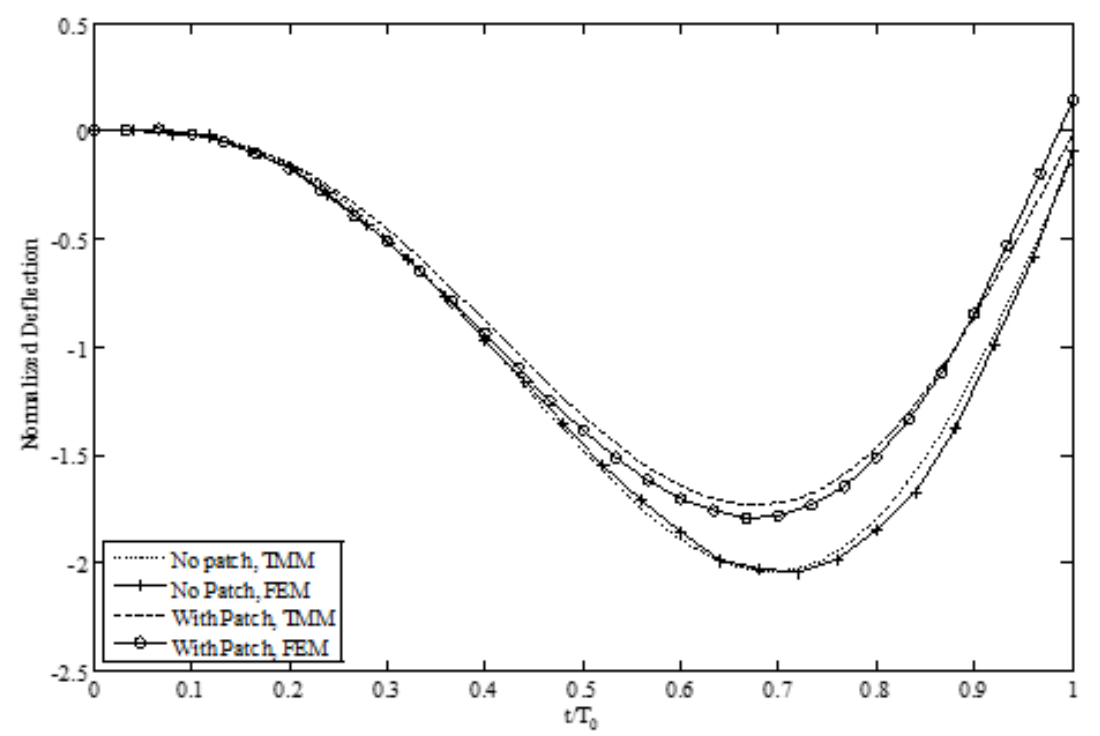

Figure 12. Comparison between mid-span displacements using composite patch. 


\section{CONCLUSIONS}

The reinforcement of a metallic beam with an edge crack at mid-span was presented in this study. A composite patch (unidirectional fiber glass/epoxy) was applied and the recovery of beam behavior at mid-span was investigated. Degradation and improvement of beam natural frequencies as the result of damage and reinforcement were studied. It was seen that increasing crack depth at mid-span has no influence on the second natural frequency. It may be because the mid-span point in the second mode shape is stationary. The length of patches were optimized in a way that the beam at mid-span behaves as like as healthy one. It was observed that using thin patch is not able to completely recover the beam deflection when the crack depth is more than $70 \%$ of beam thickness. However, thick patch could improve the beam mechanical properties with high crack depth $>70 \%$. Using thin and thick patch resulted that the most effective patch length is less than $70 \%$ of the beam's length. Stress intensity factor in the damaged beam was improved as well. It was found that applying optimum patch length for every crack depth may cause almost the same reduction ( $47 \%$ for thin patch and $75 \%$ for thick patch) in the stress intensity factor. Results of this study were obtained through programming in MATLAB software. The time history diagram of deflection for healthy, cracked and reinforced beam was verified against those which were found through FEM.

\section{REFERENCES}

[1] Alebrahim R, Sharifishourabi G, Sharifi S, Alebrahim M, Zhang Z, Yahya Y, Ayob A. Thermo-mechanical behaviour of smart composite beam under quasi-static loading. Composite Structures. 2018; 201:21-28.

[2] Alebrahim R, Haris MH, Nik Mohamed NA, Abdullah S. Vibration analysis of self-healing hybrid composite beam under moving mass. Composite Structures. 2015; 119:463-476.

[3] Alebrahim R, Haris SM, Mohamed NAN, Abdullah S. Vibration analysis of multi-cracked beam traversed by moving mass. Asian Journal of Materials Science. 2016; DOI: 10.3923/ajmskr.2016.

[4] Alebrahim R, Iqram M, Farizal M, Singh SSK, Haris SM, Elwaleed AK, Nikabdullah NM Control of crack propagation in composite fibreglass-polyester laminates using Nitinol wire. Applied Mechanics \& Materials. 2014; 663:108-112.

[5] Gherissi A. Failure study of the woven composite material: 2.5 D carbon fabric/ resin epoxy. Journal of Mechanical Engineering and Sciences. 2019; 13:5390-5406.

[6] Rayhan SB. A comprehensive study on the buckling behavior of woven composite plates with major aerospace cut outs under uniaxial loading. Journal of Mechanical Engineering and Sciences. 2019; 13:4756-4776.

[7] Shanti Kiran Z, Suresh Babu V, Soma Sekhar KVL. Study of the microhardness and erosive wear behavior of organo-modified nanoclay filled glass-epoxy composites and optimization. Journal of Mechanical Engineering and Sciences. 2019; 13:47944815 .

[8] Schubbe JJ, Mall S. Investigation of a cracked thick aluminum panel repaired with a bonded composite patch. Engineering Fracture Mechanics. 1999; 63:305-323.

[9] Chukwujekwu Okafor A, Singh N, Enemuoh UE, Rao SV. Design, analysis and performance of adhesively bonded composite patch repair of cracked aluminum aircraft panels. Composite Structures. 2005; 71:258-270.

[10] Colombi P, Panzeri N, Poggi C. Experimental characterization of steel elements reinforced by adhesively bonded CFRP plates. Advanced Polymer Composites for Structural Applications in Construction. 2004; 245-257.

[11] Megueni A, Bouiadjra BB; Boutabout B. Computation of the stress intensity factor for patched crack with bonded composite repair in pure mode II. Composite Structures. 2003; 59:415-418.

[12] Khalili SMR, Fathollahi MR. Creep analysis in smart single-strap adhesive joints reinforced by shape memory alloysExperimental study. International Journal of Adhesion and Adhesives. 2014; 54:21-29.

[13] Khalili SMR, Mittal RK, Mohammad Panah N. Analysis of fiber reinforced composite plates subjected to transverse impact in the presence of initial stresses. Composite Structures. 2007; 77: 263-268.

[14] Chue CH, Jin-Chee Liu T. The effects of laminated composite patch with different stacking sequences on bonded repair. Composites Engineering. 1995; 5:1995 223-230.

[15] Papadopoulos CA. The strain energy release approach for modeling cracks in rotors: A state of the art review. Mechanical Systems and Signal Processing. 2008; 22:763-789.

[16] Vaziri A, Nayeb-Hashemi H. Dynamic response of a repaired composite beam with an adhesively bonded patch under a harmonic peeling load. International Journal of Adhesion and Adhesives. 2006; 26:314-324.

[17] Duong CN, Yu J. An analytical estimate of thermal effects in a composite bonded repair: plane stress analysis. International Journal of Solids and Structures. 2002; 39:1003-1014.

[18] Ariaei A, Ziaei-Rad S, Ghayour M. Repair of a cracked Timoshenko beam subjected to a moving mass using piezoelectric patches. International Journal of Mechanical Sciences. 2010; 52:1074-1091.

[19] Liu TJC. Fracture mechanics and crack contact analyses of the active repair of multi-layered piezoelectric patches bonded on cracked structures. Theoretical and Applied Fracture Mechanics. 2007; 47:120-132.

[20] Alaimo A, Milazzo A, Orlando C. Numerical analysis of a piezoelectric structural health monitoring system for composite flange-skin delamination detection. Composite Structures. 2013; 100:343-355.

[21] Tada H, Paris P, Irwin G. The stress analysis of cracks Handbook, Hellertown. Pennsylvania: Del Research Corporation, 1973. 
[22] Kitipornchai S, Ke LL, Yang J, Xiang Y. Nonlinear vibration of edge cracked functionally graded Timoshenko beams. Journal of Sound and Vibration. 2009; 324: 962-982. 